\title{
Linkage mapping of a polymorphic plumage locus associated with intermorph incompatibility in the Gouldian finch (Erythrura gouldiae)
}

\author{
K-W Kim ${ }^{1}$, SC Griffith ${ }^{2}$ and T Burke ${ }^{1}$
}

Colour polymorphism is known to facilitate speciation but the genetic basis of animal pigmentation and how colour polymorphisms contribute to speciation is poorly understood. Restricted recombination may promote linkage disequilibrium between the colour locus and incompatibility genes. Genomic rearrangement and the position of relevant loci within a chromosome are important factors that influence the frequency of recombination. Therefore, it is important to know the position of the colour locus, gene order and recombination landscape of the chromosome to understand the mechanism that generates incompatibilities between morphs. Recent studies showed remarkable pre- and postzygotic incompatibilities between sympatric colour morphs of the Gouldian finch (Erythrura gouldiae), in which head feather colour is genetically determined by a single sex-linked locus, Red. We constructed a genetic map for the $Z$ chromosome of the Gouldian finch (male-specific map distance $=131 \mathrm{cM}$ ), using 618 captive-bred birds and 34 microsatellite markers, to investigate the extent of inter- and intraspecific genomic rearrangements and variation in recombination rate within the $\mathrm{Z}$ chromosome. We refined the location of the Red locus to a $~ 7.2-\mathrm{cM}$ interval in a region with a moderate recombination rate but outside the least-recombining, putative centromeric region. There was no evidence of chromosome-wide genomic rearrangements between the chromosomes carrying the red or black alleles with the current marker resolution. This work will contribute to identifying the causal gene, which will in turn enable alternative explanations for the association between incompatibility and colouration, such as fine-scale linkage disequilibrium, genomic rearrangements and pleiotropy, to be tested. Heredity (2016) 116, 409-416; doi:10.1038/hdy.2015.114; published online 20 January 2016

\section{INTRODUCTION}

Colour polymorphisms are often associated with other physiological and behavioural traits (McKinnon and Pierotti, 2010), which may generate incompatibilities between morphs, thus facilitating speciation (Hugall and Stuart-Fox, 2012; Poelstra et al., 2014). One of the explanations for these associations is pleiotropy, in which the colour polymorphism is controlled by transcription factors or hormones that influence multiple physically unlinked genes (McKinnon and Pierotti, 2010). An alternative hypothesis for the association is that there are linkage disequilibria (LD) among the loci that control these traits. Recombination is a key process that counteracts the formation of LD between loci. To date, various mechanisms that might alter the rate of recombination have been proposed, including inversion (Rieseberg, 2001), the centromere effect (Carneiro et al., 2009) and recombination hotspots (Singhal et al., 2015), which collectively generate highly variable recombination landscapes between and within species (Backström et al., 2010a; Auton et al., 2012).

For example, chromosome-wide LD caused by complex rearrangements and reduced recombination in the second chromosome of white-throated sparrows (Zonotrichia albicollis) generates the association between plumage polymorphism and social behaviour (Thorneycroft, 1966; Tuttle, 2003; Huynh et al., 2011). Similarly, the three alternative, phenotypically complex and distinctly plumaged morphs of the ruff Philomachus pugnax are determined by a large inversion and the multiple genes combined within such an inversion may act together as alleles of a supergene (Küpper et al., 2016; Lamichhaney et al., 2016).

In the zebra finch (Taeniopygia guttata; hereafter ZF), the macrochromosomes show greatly reduced recombination in the central part of each chromosome and recombination is concentrated towards the distal parts of the chromosomes, but these extreme patterns are not found in the microchromosomes (Stapley et al., 2010). In contrast, the orthologous macrochromosomes in chicken and flycatcher show moderate recombination rates in the more central regions, although there is again a tendency for recombination rates to be elevated towards the chromosome ends (Kawakami et al., 2014; Singhal et al., 2015). If a colour locus resides in a recombination desert, such as the one that spans $\sim 50 \mathrm{Mb}$ in the central region of the $\mathrm{Z}$ chromosome of ZF (Stapley et al., 2010), incompatibility loci in the same region might potentially be coupled with the colour locus. Thus, to understand the mechanism underlying any incompatibility between morphs, it is necessary to study the genomic organization and the recombination landscape of the respective chromosome, as well as locating the position of the gene that causes the polymorphism.

The Gouldian finch (Erythrura gouldiae; hereafter GF) is an ideal species in which to study colour polymorphism in relation to incompatibility between morphs. The GF belongs to the Estrildidae, the same family as ZF, and is estimated to have separated from ZF 12.8 million years ago (Jetz et al., 2012). This was one of the first avian species in which a plumage colour polymorphism was studied using

${ }^{1}$ Department of Animal and Plant Sciences, University of Sheffield, Sheffield, UK and ${ }^{2}$ Department of Biological Sciences, Macquarie University, Sydney, NSW, Australia Correspondence: Dr K-W Kim, Department of Animal and Plant Sciences, University of Sheffield, Western Bank, Sheffield S10 2TN, UK.

E-mail: kangwook.kim.ego@gmail.com

Received 9 July 2015; revised 25 November 2015; accepted 11 December 2015; published online 20 January 2016 
classical genetics (Southern, 1945). A single sex-linked Mendelian locus (hereafter, the Red locus) with dominant red $\left(Z^{R}\right)$ and recessive black $\left(Z^{r}\right)$ alleles determines the expression of head colour (Southern, 1945). In females, the heterogametic sex in birds, the phenotype matches the hemizygous genotype $\left(Z^{R}\right.$, red; $Z^{r}$, black), whereas males can be homozygous $Z^{r} Z^{r}$ (black), $Z^{R} Z^{R}$ (red) or heterozygous $Z^{R} Z^{r}$ (red). This polymorphic trait attracts special attention because the sex chromosome is expected to have a disproportionately large influence on the speciation process, either by selection or drift (Qvarnström and Bailey, 2008; Mank et al., 2010), and colour polymorphisms are often subject to sexual selection (Wellenreuther et al., 2014). Moreover, the Red locus has been shown to be associated with both pre- and postzygotic incompatibilities between morphs in a captive population (Pryke and Griffith, 2007, 2009b; Pryke, 2010). However, it has not been confirmed whether incompatibility occurs in the wild population and a lack of knowledge on the genetic architecture of the polymorphism hampers our understanding of the underlying cause of the reported incompatibilities.

In the present study, we examined two large-scale factors that might potentially cause the genetic incompatibility associated with head colour by constructing a genetic map for the $\mathrm{Z}$ chromosome of the GF and localizing the Red locus on the map. First, we examined whether the Red locus is located in a genomic region with reduced recombination, such as the centromere or a large recombination desert as is found in the ZF. Second, we compared the gene order between $\mathrm{Z}$ chromosomes containing the alternative alleles at the Red locus to test for genomic rearrangements between chromosomes determining the two morphs.

\section{MATERIALS AND METHODS}

\section{Samples}

Blood samples $(n=618)$ were collected from a captive population that was originally established with wild-type, but domesticated, birds from 23 aviculturists throughout Australia (Pryke and Griffith, 2006) and stored in 100\% ethanol at room temperature before DNA extraction using the ammoniumacetate precipitation method (Bruford et al., 1998).

\section{Marker development and genotyping}

Previously, we developed a large number of microsatellite markers for GF in various ways (Kim et al., 2015), including Sanger and 454 sequencing of microsatellite-enriched libraries, in silico primer design based on ZF microsatellite sequences found in the reference genome, Taeniopygia_guttata-3.2.4 (Warren et al., 2010) and testing microsatellite primers designed in other species (Supplementary Table S1). In total, 39 sex-linked markers were selected and multiplex sets were developed and tested for genotyping. The flanking sequences of the cross-species and library-derived microsatellites were used to search for the physical positions of markers in the ZF genome assembly with the blastn option (e-value $<\mathrm{e}-10)$ in a locally installed BLAST program.

DNA ( $20 \mathrm{ng}$ ) was dried down in PCR plates in preparation for the PCR reaction. Two-microlitre PCR reactions were set up containing $1 \mu \mathrm{l}$ of Qiagen Multiplex PCR Kit (Qiagen, Hilden, Germany) and $1 \mu \mathrm{l}$ of diluted primers $(0.2 \mu \mathrm{M}$ each). PCR amplification was performed using DNA Engine Tetrad PTC-225 Peltier Thermal Cyclers (MJ Research, Bio-Rad, Hercules, CA, USA). PCR products were separated on an ABI 3730 DNA Analyser (Applied Biosystems, Waltham, MA, USA) and genotypes were scored using GENEMAPPER v.3.7 (Applied Biosystems).

For the sequencing, PCR products were first cleaned with ExoSAP-IT (Affymetrix, Santa Clara, CA, USA). After the sequencing reaction was completed using a BigDye Terminator v.3.1 Cycle Sequencing Kit (Applied Biosystems), and the reaction products precipitated using the ethanol/EDTA method, the products were separated on an ABI 3730 DNA Analyser. Sequences were aligned and edited using CodonCode Aligner v. 2.0.6 (CodonCode Corporation, Centerville, MA, USA).

\section{Genotype error correction}

A Perl program (CriMaker; http://dx.doi.org/10.5061/dryad.fg7g4) was developed to compile the genotypic data into CRI-MAP format and to identify inconsistencies in the Mendelian inheritance of markers. Initial error checking revealed 1804 genotypic mismatches between parents and offspring, but these were caused mostly by a few individuals who were concluded to have false pedigree information (the birds had been bred in aviaries containing social groups, thus some misidentification of parents was possible), and some errorprone markers. By removing 27 individuals who caused errors across the markers, and four markers that caused errors across families, the number of errors dropped to 245. These errors consisted of inherited null alleles, 1-bp binning mismatches or rare mutations, and all could be corrected manually by comparing the pedigree and genotypes. The remaining 34 loci were used in the subsequent analysis (Supplementary Table S1).

\section{Construction of Z-chromosome genetic map and linkage mapping} of the Red locus

Two- or three-generation half-sib families in which 73 fathers each mated with 1-4 females were used to construct the genetic linkage map of the $\mathrm{Z}$ chromosome. If a parent's DNA was not available, then genotypes were, whenever possible, inferred from the offspring's genotypes. The genotype of the mother (ZW) could be inferred with certainty because a maternal allele on the $\mathrm{Z}$ chromosome should be seen in all sons but not in daughters, whereas the paternal alleles could be transmitted to both sons and daughters. The sex of each individual was determined with two sex-linked markers, Z-002D (Dawson, 2007) and $Z 37 B$ (Dawson et al., 2015), that each amplified alleles on both the $Z$ and $\mathrm{W}$ chromosomes. The results agreed perfectly with other markers that showed only a single allele in females and one or two alleles in homozygous and heterozygous males, respectively. Pedigree information and the head colour after moulting were recorded, but because of a high mortality rate in mixedmorph breeding of offspring from embryos up to the age at which the trait is expressed in 6- to 9-month-old juveniles (Pryke and Griffith, 2009b), only $65.4 \%$ of samples had phenotypic data available (404/618). The mother's genotype at the colour locus could be inferred directly from their phenotype, whereas the genotype of red-headed males had to be inferred from the pedigree information. For example, a black male (always $Z^{r} Z^{r}$ ) breeding with a red female $\left(Z^{R}\right)$ will produce only red heterozygous male offspring, whereas a heterozygous red male $\left(Z^{R} Z^{r}\right)$ breeding with a black or red female $\left(Z^{r}\right)$ can produce both red and black offspring.

To determine the marker order and intervals between markers, a maximumlikelihood linkage analysis was performed using CRI-MAP (Green et al., 1990) as improved by Evans and Maddox (v.2.503, http://www.animalgenome.org/ tools/share/crimap/). First, a framework marker map was created using the option build and the positions of the remaining markers were determined with the option all, thus adding one marker at a time. If this option gave two or more positions with nonsignificant difference in the $\log _{10}$ likelihood $(<3)$ between positions, the order in the ZF reference genome was considered to be the most likely one. This order was then tested with options flips2, flips3 and flips 4 to see if there was a better order, and an increase in $\log _{10}$ likelihood of three or more was taken as evidence of a significantly more likely map (Morton, 1955). None of these tests produced a better order than the original. Undetected errors that introduce less-likely double recombinants were examined with the option chrompic and the map was recreated after correction, as described above.

In birds, recombination on the sex chromosome (other than in the pseudoautosomal regions) occurs only in the male, thus the effective recombination rate on the $\mathrm{Z}$ chromosome should be $\sim 2 / 3$ of the malespecific estimate (Bennett and Oertel, 1965). Caution is required when using CRI-MAP as it calculates the sex-averaged recombination rates on the sex chromosome as being half of the male-specific rate. We report male-specific maps with Kosambi map distances to account for meiosis only in the father and to compare with previous literature that used male-specific rates, but these values can be easily converted to sex-averaged rates by multiplying by $2 / 3$. The maps were drawn using MapChart v.2.2 (Voorrips, 2002).

In total, 11 families were informative for segregation at the Red locus and used to obtain two-point LOD scores (logarithm (base 10) of odds) between 
the colour locus and genetic markers. These families were either fully informative crosses between a heterozygous red male $\left(Z^{R} Z^{r}\right)$ and a black female $\left(Z^{r}\right)$ or partially informative crosses between a heterozygous red male and a red female $\left(Z^{R}\right)$, where only female offsprings are informative because all male offsprings will have the red phenotype. The best position for the Red locus was searched for by comparing the likelihoods of alternative map positions of the Red locus using the all function of CRI-MAP and an increase of three or more in $\log _{10}$ likelihoods was considered as evidence of a significantly more likely map position.

\section{Intraspecific genomic rearrangement}

To test for intraspecific genomic rearrangements between morphs, the abovedescribed mapping families were subdivided into three subsets according to the father's genotype at the colour locus based on pedigree information across several generations and their phenotypes: first, homozygous black fathers $(n=31)$ who had two 'black chromosomes' (the chromosome that has the recessive black allele $Z^{r}$ ); second, homozygous red fathers $(n=15)$ who had two 'red chromosomes' (the chromosome that has the dominant red allele $Z^{R}$ ); and third, heterozygous red fathers $(n=17)$ who had both black and red chromosomes. Genotypes of 10 fathers in the mapping families could not be determined because of the limited pedigree information we had for them and these were excluded from this analysis. Using these subsets, three separate maps were constructed and the marker orders were compared with each other.

\section{RESULTS}

Linkage map for the Gouldian finch Z-chromosome and intrachromosomal rearrangements between species

A total of 73 families were genotyped with 34 microsatellite markers (Supplementary Table S1), giving 582 informative crossovers. First, we created a framework marker map composed of 28 markers and determined the positions of six remaining markers to obtain the bestorder map; this had a mean intermarker spacing of $4 \mathrm{cM}(0-31.9 \mathrm{cM})$ and a total male-specific map length of $131 \mathrm{cM}$ (Supplementary Figure S1 and Figure 1a). This map corresponded to $97.2 \%$ of the physical map length of the $\mathrm{ZF} \mathrm{Z}$ chromosome, assuming that the physical size of the $\mathrm{Z}$ chromosome is conserved in both species because the same terminal markers in GF (TguE103 and TguE157) were also located at the ends of the chromosome in ZF (Figure 1a).

Genetic map positions of the GF microsatellite markers were compared with the physical map position on the ZF reference genome a

$$
\begin{aligned}
& \text { Zebra finch } \\
& \text { physical map }
\end{aligned}
$$

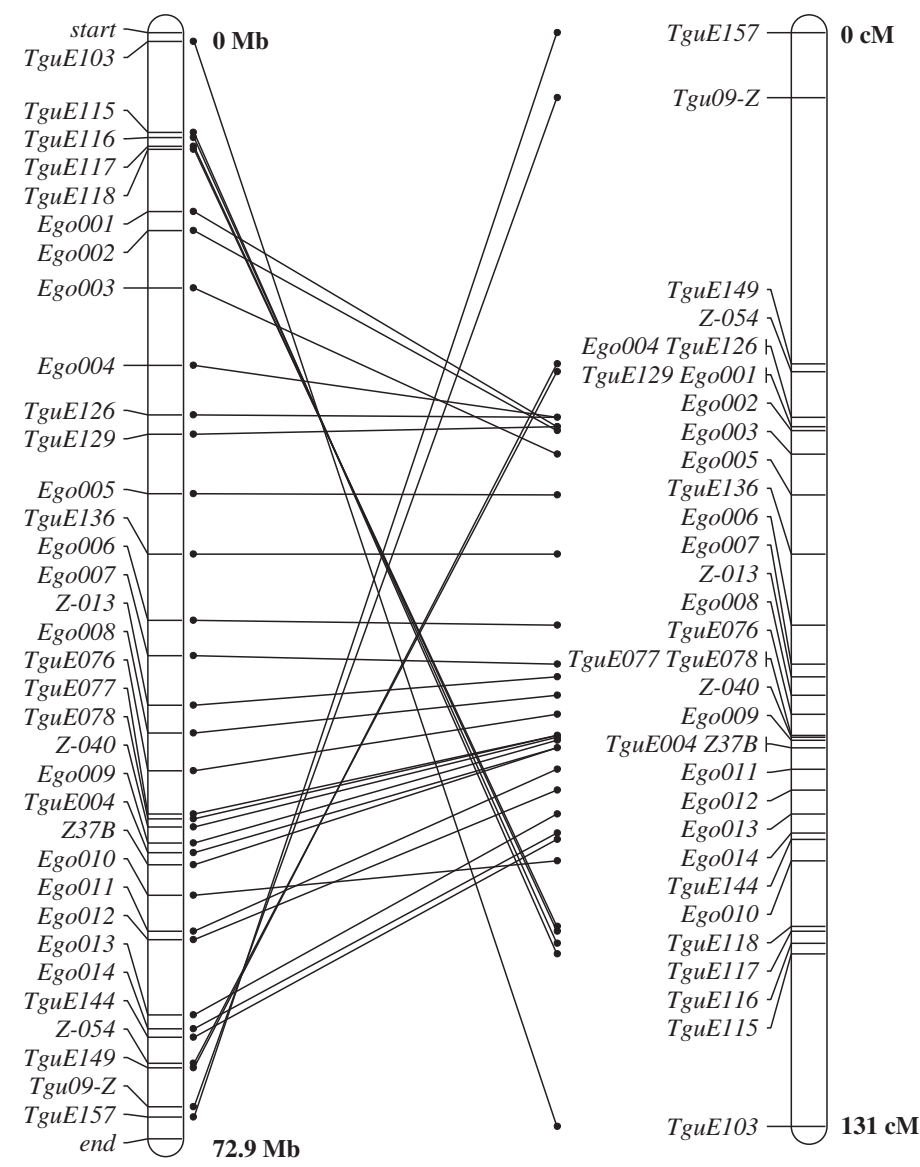

b

Zebra finch physical map

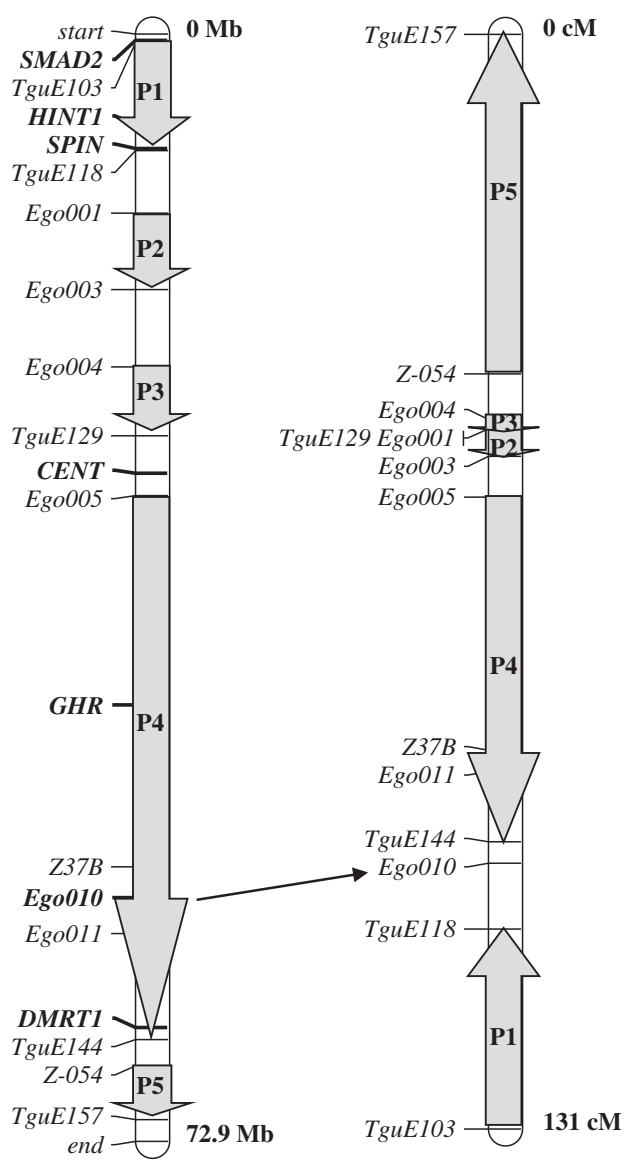

Figure 1 Interchromosomal rearrangements between zebra finch and the Gouldian finch. (a) The best-order genetic map positions of 34 microsatellite markers in the GF Z chromosome are compared with the physical map positions in ZF, with straight lines connecting identical marker positions. (b) Schematic overview of the interchromosomal rearrangements between the two species. The orientations of five chromosomal regions (P1-P5) that share an identical marker order are indicated by arrows. The positions of the putative centromere (CENT) and five genes in ZF are based on Itoh et al. (2011). The GF map is oriented to illustrate the collinear relationship between the two species in the P4 region, which has the biggest predicted physical length. In addition, it is more likely that our illustration represents the order in the submetacentric karyotype of GF, given the relative size of each region and the predicted position of the centromere in GF. 
assembly (Warren et al., 2010). Various degrees of intrachromosomal rearrangement were observed between the two species and these resulted from changes in the position of five chromosomal fragments that shared the same marker order between the two species (P1-P5) (Figure 1b). First, a major pericentric inversion including the region between markers Ego001 and TguE144 interchanged the positions of both telomeric regions ( $\mathrm{P} 1$ and $\mathrm{P} 5)$ in relation to the $\mathrm{P} 4$ region. Second, a small fraction of the chromosome, including only one marker (Ego010), showed a pattern of intrachromosomal transposition (Figure 1b). To verify and define the range of an intrachromosomal translocation of this locus, primers for 11 introns of the genes flanking marker Ego010, between markers Z37B and Ego011, were tested for sequencing. Informative SNPs that were obtained from 24 individuals in 5 families using four amplifying primers showed that a chromosomal fragment with a size of up to $3.5 \mathrm{Mb}$ had changed its position in GF compared with ZF (Supplementary Table S2 and Supplementary Figure S2). Third, the positions of chromosomal sections P2 and P3 were different in the two species (ZF and GF). This could not be explained by a simple inversion because the gene order within each fragment was the same as in $\mathrm{ZF}$, and the orientations of the fragments were unchanged (Figure 1b). However, the marker order in these regions was not resolved well because of the small number of informative crossovers.

Despite large-scale genomic rearrangements between species, marker orders within chromosomal fragments were conserved between the two species. For example, the P4 region, the largest chromosomal fragment in the long (q)-arm spanning $35.8 \mathrm{Mb}$ in $\mathrm{ZF}$ between Ego005 and TguE144, showed almost the same order of markers, except for EgoO10 (Figure 1a).

\section{The rate of recombination}

The overall recombination rate of the GF $\mathrm{Z}$ chromosome was estimated by comparing the genetic distance between the endmost markers in the GF $\mathrm{Z}$ chromosome with the physical distance of the corresponding positions of the ZF Z chromosome. Assuming a similar physical size of the $Z$ chromosome in the two species, the estimated physical distance between the endmost markers (TguE157 and TguE103) in GF was about $70.9 \mathrm{Mb}$ and the overall recombination rate was $1.85(131 / 70.9) \mathrm{cM} \mathrm{Mb}^{-1}$.
Linear regression of GF linkage map position against the ZF physical map position was performed for each chromosomal fragment to obtain the slopes as estimates of regional recombination rates. There was considerable local variation in the rate of recombination among five chromosomal fragments (Figure 2). High recombination rates were found in the terminal sections: 3.4 and $11.8 \mathrm{cM} \mathrm{Mb}^{-1}$ in $\mathrm{P} 1$ and $\mathrm{P} 5$, respectively. In particular, the P5 region, spanning just $3.5 \mathrm{Mb}$ in $\mathrm{ZF}$, occupied almost a quarter of the genetic distance of the GF genetic map.

In contrast, the $\mathrm{P} 2$ and $\mathrm{P} 3$ regions showed low rates of recombination. These regions, spanning $14.7 \mathrm{Mb}$ of the short (p)-arm in $\mathrm{ZF}$, corresponded to a small genetic distance $(4.4 \mathrm{cM})$ in $\mathrm{GF}$, resulting in low recombination rates: 0.7 and $0.2 \mathrm{cM} \mathrm{Mb}^{-1}$ in $\mathrm{P} 2$ and $\mathrm{P} 3$, respectively. This, in turn, suggests that these regions may be close to the centromere. The region spanning the greatest physical length in ZF, P4, showed an intermediate recombination rate in $\mathrm{GF}\left(1 \mathrm{cM} \mathrm{Mb}^{-1}\right.$, excluding a translocated marker, Ego010; Figure 2).

\section{Linkage mapping of the Red locus}

Linkage between the colour locus and genetic markers was examined with male-specific two-point LOD scores (Figure 3). The markers (Ego008-TguE78) within the region between Z-013 and Z-040, which spanned $7.2 \mathrm{cM}(\sim 8 \mathrm{Mb}$ in $\mathrm{ZF})$, showed perfect cosegregation with the phenotype (i.e. the recombination fractions between these markers and the colour locus were zero). These markers showed the highest LOD score for linkage (two-point $\mathrm{LOD}=16.3$; Figure 3 ). The likelihood of the map position of the Red locus was equally high across this range, whereas any other map position for the Red locus resulted in a significant decrease $(>3.3)$ in the $\log _{10}$ likelihood of the map. The Red locus was therefore mapped to the centre of the P4 region, and this candidate region showed an intermediate recombination rate $\left(0.9 \mathrm{cM} \mathrm{Mb}^{-1}\right.$; Figure 2).

\section{Intrachromosomal rearrangement between morphs}

To examine if there were genomic rearrangements between chromosomes carrying each colour allele, linkage maps for three subsets of families that each had fathers with one of the three combinations of Black- and Red-determining chromosomes (Black x Black, Black x Red and Red x Red) were constructed and the sizes of the linkage maps were $131.7,128.1$ and $123.7 \mathrm{cM}$,

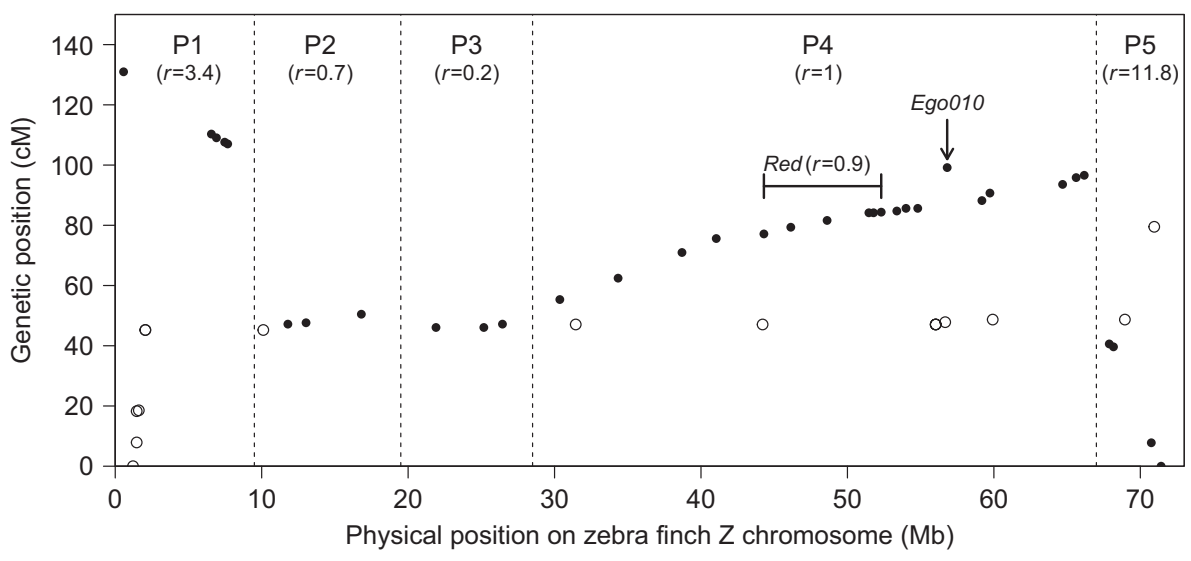

Figure 2 Linkage map position (cM) plotted against the predicted position in the zebra finch (Mb) for the Gouldian finch (filled circles) and zebra finch (open circles: data courtesy of Stapley et al., 2010). Vertical dotted lines divide the chromosome into five sections (P1-P5; see text for details), each of which has similarity with the respective chromosomal section in the zebra finch, with the most likely order in GF being P5-P3-P2-P4-P1 (Figure 1). The horizontal bar (Z-013-Z-040) shows the candidate region that contains the Red locus (see Figure 3); $r$, the absolute linear regression slope within each fragment represents the regional recombination rate $\left(\mathrm{cM} \mathrm{Mb}{ }^{-1}\right)$. 
respectively (Supplementary Figure S3 and Figure 4). If a chromosomal polymorphism were present, then it would be expected to produce different genetic maps from fathers with Black x Black and
Red $\mathrm{x}$ Red chromosomes. In addition, the map from Black $\mathrm{x}$ Red would be expected to show reduced recombination in the rearranged region. The size of the linkage map was smallest in

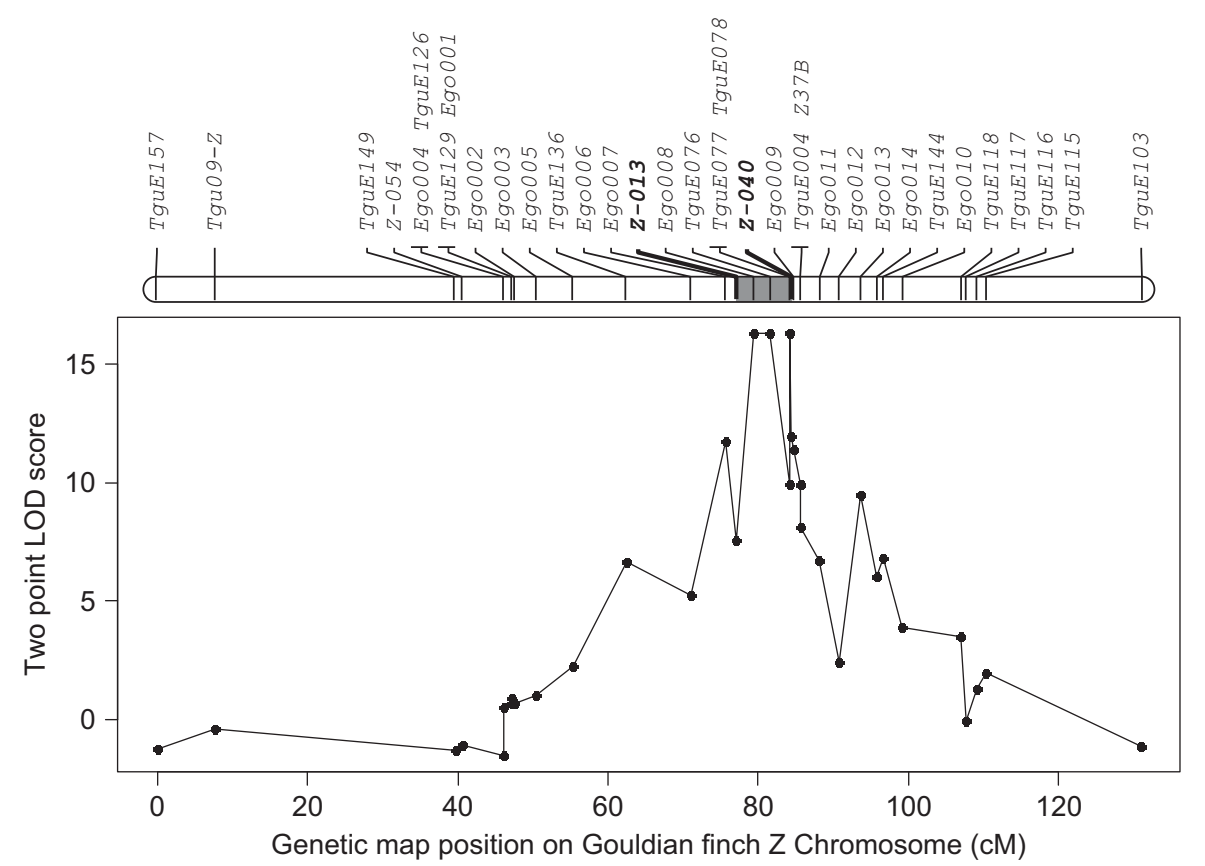

Figure 3 Male-specific two-point LOD score profiles for linkage between the Red locus and microsatellite markers plotted against Z-chromosome genetic map position in the Gouldian finch. The interval that is most likely to contain the Red locus is shaded in grey between Z-013 and Z-040.

Black x Black

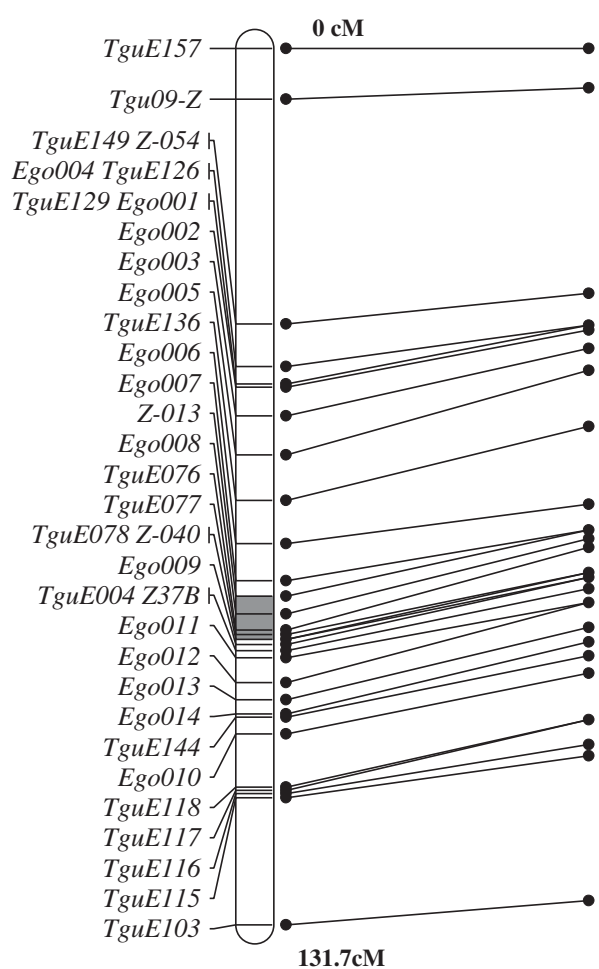

Black x Red

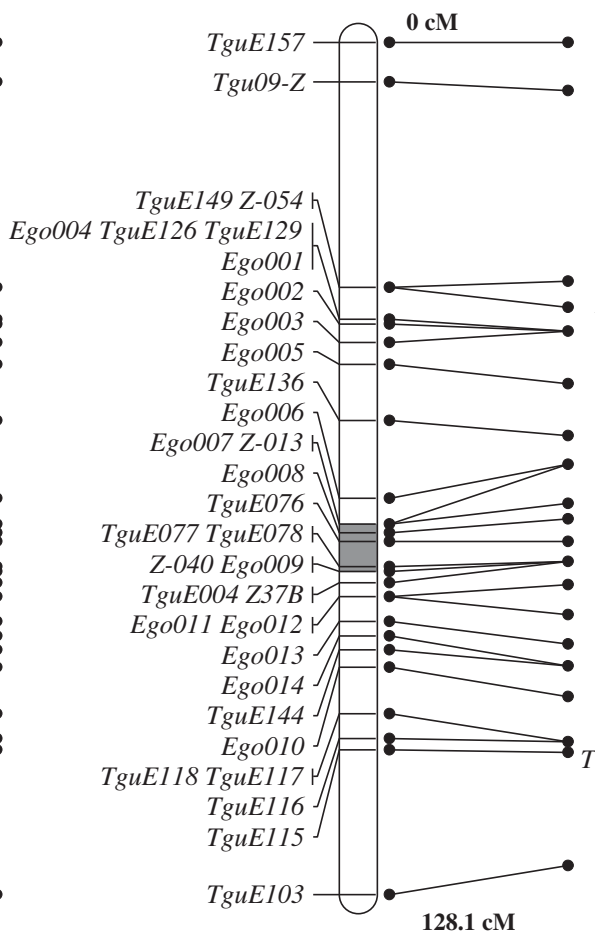

Red x Red

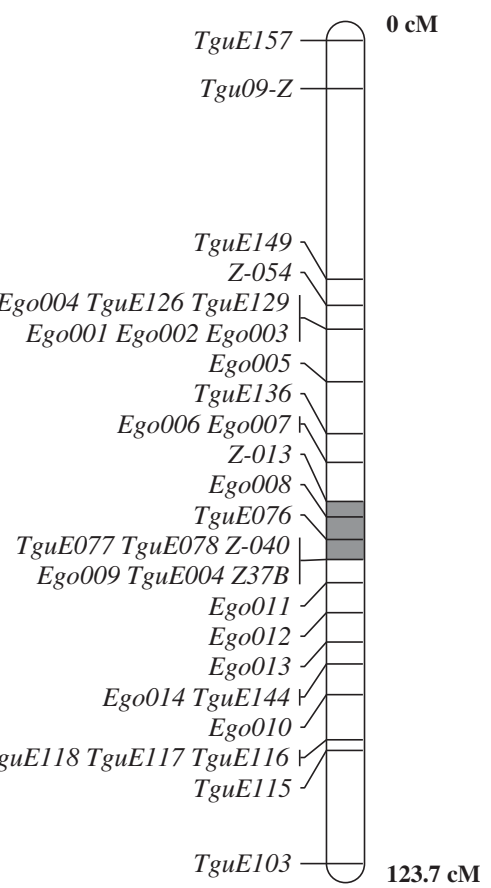

Figure 4 Comparative best-order genetic maps of the markers from three types of father, with different combinations of Black and Red chromosomes, in relation to the genotype at the Red locus: Black x Black, Red $x$ Red and Black $x$ Red, where the Black and Red chromosomes denote the chromosomes that have a recessive black allele $\left(Z^{\prime}\right)$ or the red allele $\left(Z^{R}\right)$, respectively. The father's genotypes were inferred from the pedigree and the phenotypes of their offspring. The shaded areas in the bar show the most likely map position of the Red locus in the $\mathrm{Z}$ chromosome. 
the Red $\mathrm{x}$ Red chromosome combination, but it was not possible to conclude that this is a property of this combination because the number of families used for the mapping of this type was smallest, allowing few observations of crossovers. Overall, there was no evidence of changes in marker order, suggesting no detectable intraspecific genomic rearrangements around the Red locus at the resolution provided by the current marker density.

\section{DISCUSSION}

\section{Genomic rearrangements between species}

Information on genomic organization is essential for mapping a focal trait on a chromosome and for studying the effect of genomic location on the trait. Many avian species share highly conserved synteny, but with various degrees of intrachromosomal rearrangement (Kawakami et al., 2014; Zhang et al., 2014). For example, no genomic rearrangements were found in the sex chromosomes between closely related Ficedula flycatcher species (Backström et al., 2010b), whereas there are karyotypic polymorphisms on the $\mathrm{Z}$ chromosome in $\mathrm{ZF}$ (Itoh et al., 2011) and an inversion is associated with alternative reproductive morphs in the ruff (Küpper et al., 2016; Lamichhaney et al., 2016). Estrildid finches have experienced several genomic rearrangements in the process of speciation. An estimation based on karyotypic banding patterns over part of this phylogenetic branch suggest that an inversion has been fixed every 2.26 My in the estrildid finches (Hooper and Price, 2015). Within the genus Erythrura, for example, the blue-faced parrotfinch (Erythrura trichroa) is separated from GF by two autosomal inversions (Christidis, 1986b; Hooper and Price, 2015). Christidis (1986a) described karyotypic polymorphism in the ZF Z chromosome based on early cytological and banding techniques. Recently, Itoh et al. (2011) rediscovered these withinspecies genomic rearrangements and mapped the relative positions of some genes by using fluorescent in situ hybridization (FISH) mapping (Itoh et al., 2006, 2011), which showed that the current ZF reference genome sequence was based on a submetacentric $Z$ chromosome, whereas an alternative metacentric $\mathrm{Z}$ chromosome has been created by an inversion.

The Gouldian finch is known to have a submetacentric $\mathrm{Z}$ chromosome (Christidis, 1986b). Although it is not possible to determine which marker should be placed at the top of the map using a genetic map alone, our illustration (Figure 1) shows the most likely orientation of the submetacentric Z chromosome of GF under the conventional definition of chromosome arms, with the P5 region belonging to the $\mathrm{p}$ arm and $\mathrm{P} 1$ region being in the $\mathrm{q}$ arm, for the following reasons. First, although the precise position of the centromere of GF needs verification, we can predict the approximate position of the centromere based on the recombination rate. The order of fragments P2 and P3 in the GF best-order map appears to have been changed compared with $\mathrm{ZF}$, but this cannot be explained by a simple inversion because the orientation of the markers inside the fragments was unchanged. It is likely that the marker order in this region has not been fully resolved because only a few informative crossovers were available because of the low recombination rate, which in turn suggests that the GF centromere may be close to this region. Moreover, the predicted physical size of $\mathrm{P} 4$ plus $\mathrm{P} 1$ is greater than the combined size of the P5, P3 and P2 regions, suggesting that the GF $\mathrm{q}$ arm may consist of the $\mathrm{P} 4$ and $\mathrm{P} 1$ regions. Interestingly, when the relative position of the centromere in submetacentric $\mathrm{Z}$ chromosome of ZF, which was shown to be at $\sim 40 \%$ of the chromosomal length from the $\mathrm{p}$ terminus (Itoh et al., 2011), was applied to the reference sequence of the $\mathrm{ZF} \mathrm{Z}$ chromosome, the location of the centromere in ZF was predicted to be between TguE129 and Ego005 in
ZF (Figure 1b). This location is close to the $\mathrm{P} 3$ region, which we found to have the lowest recombination rate in GF (Figure 2).

A comparison between $\mathrm{ZF}$ and GF shows that a pericentric inversion has changed the relative locations of the terminal regions of the $\mathrm{Z}$ chromosome. To examine the changes in gene order in relation to the major inversion between the GF and ZF Z-chromosome karyotypes, the positions of five genes that were used in the previous study (Itoh et al., 2011) were compared with the relative positions of the microsatellite markers used in this study (Figure 1b). Assuming no gene order changes within the five predefined fragments, the chromosomal fragment that contains GHR (growth hormone receptor) and DMRT1 (doublesex and mab-3 related transcription factor 1) was found on the $\mathrm{q}$ arm in GF and in both chromosomal variants of ZF. In ZF, SMAD2 (mothers against DPP homologue 2), HINT1 (histidine triad nucleotide binding protein 1) and SPIN (spindlin) were found in the $\mathrm{p}$ arm of submetacentric $\mathrm{Z}$ chromosome, whereas $S M A D 2$ and the major part of an HINT1-containing BAC probe were mapped onto the $\mathrm{q}$ arm, and SPIN was found in the $\mathrm{p}$ arm in metacentric Z chromosome (Itoh et al., 2011). In GF, however, the chromosomal fragment P1 (between TguE103 and TguE118) that may contain HINT1, SPIN and possibly SMAD2 (distance between SMAD2 and $\operatorname{TguE103}$ is $122 \mathrm{~kb}$ in $\mathrm{ZF}$ ), was mapped onto the $\mathrm{q}$ arm. Therefore, neither of the chromosomal polymorphisms in ZF had the same gene order as GF, suggesting that the inversions in the two species had different origins.

We also found evidence for an intrachromosomal translocation that has occurred within a genomic region around EgoO10 (maximum $3.5 \mathrm{Mb}$ ), although this could have been derived from more complex genomic rearrangements (Supplementary Table S2 and Supplementary Figure S2). This region provides a good model for the future study of the relationship between genomic rearrangements and sequence evolution because protein-coding sequences may evolve faster in chromosomes that have undergone structural rearrangements (Navarro and Barton, 2003). Currently, the position of Ego010 within the GF genome is not supported very well because of the sparse marker density between the $\mathrm{P} 1$ and $\mathrm{P} 4$ regions. It is possible that the change in genomic position may also have influenced the recombination rate in the translocated fragment because it was moved closer to the end of the chromosome, where the recombination rate is elevated. Interestingly, the translocated region contained a putative pigmentation gene, proteinase-activated receptor 2 precursor (PAR-2 or F2RL1), that upregulates melanin and melanosome ingestion by increasing keratinocyte phagocytosis (Sharlow et al., 2000). Therefore, further studies in this region may provide an insight into the relative roles of selection and genomic rearrangements in the evolution of pigmentation genes.

\section{Variation in recombination rate}

Some researchers assume similar recombination landscapes across confamilial species (Hooper and Price, 2015). However, our results indicate that finch species in the same family can have very different recombination landscapes. In the $\mathrm{ZF}$, an exceptionally biased recombination landscape is observed, in which the majority of recombination occurs in the distal regions, whereas large recombination deserts are found in the central parts of the macrochromosomes (Kawakami et al., 2014; Singhal et al., 2015). For example, the three largest chromosomes (TGU1-TGU3) have large recombination deserts spanning over $100 \mathrm{Mb}$, with a mean recombination rate of $0.1 \mathrm{cM} \mathrm{Mb}^{-1}$, and the $\mathrm{Z}$ chromosome shows an extremely low recombination rate in the central part, causing significant LD across a 50-Mb region (Backström et al., 2010a; Stapley et al., 2010). 
In the present study, we examined if this pattern was also found in GF. Indeed, a highly heterogeneous recombination rate was again found within the $\mathrm{Z}$ chromosome but the pattern was different to that of $\mathrm{ZF}$. Based on the recombination rate and a comparison with ZF, we predicted that the GF centromere might be located close to the P3 $\left(0.2 \mathrm{cM} \mathrm{Mb}^{-1}\right)$ region. However, the reduced recombination did not extend beyond this region. In contrast, both termini of the chromosome showed greatly increased recombination rates that account for a large fraction of the entire genetic map, consistent with other avian species (Kawakami et al., 2014). The P4 region that has the largest collinear gene order compared with ZF showed an intermediate level of recombination.

The mechanism that causes this variation in recombination rate is not clearly understood, but chromosome size, GC content and sequence motif have been proposed to be important factors (Kawakami et al., 2014; Singhal et al., 2015). In this study, direct comparison of genomic sequences and recombination rates between these species was not possible because of the non-availability of a genome sequence for the GF. An alternative mechanism that might cause the differences in the rate of recombination might be the described genomic rearrangement, because such a major pericentric inversion as that seen between ZF and GF would have changed the relative positions of many loci within the chromosome.

\section{Colour-gene mapping}

The extent of genomic rearrangements and the rate of recombination provide crucial information for gene mapping. The conserved marker order in the $\mathrm{P} 4$ section of the $Z$ chromosome, when compared with $\mathrm{ZF}$, allows us to infer that gene order is well conserved in this area. If a candidate locus were found in this region, then it should be possible to refer to the well-annotated ZF genome databases to obtain a list of candidate genes and predict their function. However, as can be seen in the transposition of locus Ego010, it is also possible that some small regions have changed their position, such that they cannot be detected with the current density of markers.

The striking differences in the rate of recombination between distal and central regions of the chromosome indicate that different strategies might be needed in different regions of the chromosome to identify the location of a target gene. First, more markers should be added in the distal regions to increase the resolution of recombination mapping. Second, more informative mapping families are required to resolve the high LD between markers in the central part of the $\mathrm{Z}$ chromosome. However, these options are not always available and are sometimes impractical. We concluded that the Red locus is in the P4 region, where the local recombination rate is about $0.9 \mathrm{cM} \mathrm{Mb}^{-1}$. Thus, thousands of meioses might be required to observe enough informative recombinant events to map the locus more finely to a $<1 \mathrm{Mb}$ region. In the GF it is also likely to be difficult to obtain enough samples that include adequate phenotypic information, because informative crosses between heterozygous red males and black females produce offspring of low viability in this captive population (Pryke and Griffith, 2009a, b). Therefore, it would be difficult to obtain finer resolution than the currently identified candidate region from linkage mapping; a genomewide association study using many more markers and wild population samples that have small $\mathrm{LD}$ is required to help narrow down the candidate region (Johnston et al., 2011).

\section{Colour locus and mating incompatibilities}

In the captive GF population also studied here, the Red locus was shown to be associated with pre- and postzygotic reproductive incompatibilities (Pryke and Griffith, 2007, 2009b). In theory, the observed association may be caused either by LD between the Red locus and incompatibility genes or a pleiotropic effect of the Red locus.

In the present study, we mainly focused on two large-scale factors that might generate high LD between the Red locus and incompatibility genes. First, we examined local recombination around the Red locus. Usually, the recombination rate in the centromeric region is significantly reduced, which often results in faster genetic differentiation between diverging species (Stump et al., 2005; Carneiro et al., 2009). We identified a putative centromeric region where the recombination rate was greatly reduced below that of any other region, but this region was much smaller than the recombination desert in the Z chromosome in ZF (Backström et al., 2010a; Singhal et al., 2015). The Red locus was mapped to a region with a moderate recombination rate, far from the putative centromere region, suggesting that the Red locus was not influenced by a centromere effect.

Second, we examined the possibility of the intermorph rearrangement, such as an inversion, which may lead to genetic differentiation by reducing the fitness of heterozygotes or by reducing gene flow through the suppression of recombination (Rieseberg, 2001). We could not find any evidence of a large-scale genomic rearrangement between morphs and the marker order was highly conserved among the three maps that were constructed from different combinations of chromosomes. However, this result does not necessarily exclude the possibility of genomic rearrangement at a finer scale. For example, if a small-scale inversion were present in the region of low recombination, or in the regions between the currently typed markers, then it would have been difficult to detect by linkage mapping without a much larger sample size.

Although not tested in this study, LD may be higher in the captive population than wild populations due to reduced effective population size and inbreeding. In particular, it is worth noting that there might have been artificial selection in captive populations in favour of high-quality head colouration (Evans and Fidler, 2005), or to produce particular colour morphs, by breeding them separately, and thus driving genetic structuring in the domesticated population. Therefore, we need to verify whether the incompatibility is a captive population-specific phenomenon, with the increased LD coupling the Red locus with incompatibility genes.

Alternatively, pleiotropy may have had an important role in the association of colour locus with incompatibility. In vertebrates, several genes involved in melanin-based colouration (e.g. Proopiomelanocortin) have been reported to be associated with sexual behaviour, aggressiveness, resistance to stressors, immune response and metabolic rate through pleiotropic effects (Ducrest et al., 2008). The red/black colour polymorphism in the Gouldian finch is mainly due to a difference in the pigments that are deposited in the feathers (Brush and Seifried, 1968). The black morphs deposit melanin, whereas red morphs deposit carotenoid in the feathers of the head. The gene(s) involved in pigment production or deposition may regulate very heterogeneous processes, which may in turn generate the incompatibility between morphs.

To conclude, there was no evidence of a large-scale reduction in recombination around the Red locus. In addition, we showed pronounced between- and within-species variation in the recombination rate within the $\mathrm{Z}$ chromosome. However, the Red locus was mapped to a region with a moderate degree of recombination that is greater than in the putative centromeric region, which showed the lowest recombination rate. Although there were various degrees of chromosomal rearrangement in comparison with ZF, no genomic rearrangements between the chromosomes that contain polymorphic colour alleles were detected with the current marker density. 
To investigate the cause of incompatibilities in the Gouldian finch, including the possibilities of a locally reduced recombination rate, fine-scale genomic rearrangements and pleiotropy, it will be necessary to identify the causal gene for the colour polymorphism using a higher-density marker approach. Our identification of the position of the Red locus will assist in targeting such efforts and revealing the genetic basis of the colour polymorphism.

\section{DATA ARCHIVING}

CriMaker and Microsatellite genotypes available from the Dryad Digital Repository: http://dx.doi.org/10.5061/dryad.fg7g4.

\section{CONFLICT OF INTEREST}

The authors declare no conflict of interest.

\section{ACKNOWLEDGEMENTS}

This study was supported by funding from the University of Sheffield for a studentship to K-WK, and from the Australian Research Council to SCG and TB. We are grateful to Sarah Pryke and the Save the Gouldian Fund for providing access to samples. Compliance with Ethical Standards: Samples: Blood sampling of birds was conducted under the approval of the Animal Ethics Committees at the University of New South Wales and Macquarie University.

Auton A, Fledel-Alon A, Pfeifer S, Venn O, Ségurel L, Street T et al. (2012). A fine-scale chimpanzee genetic map from population sequencing. Science 336: 193-198.

Backström N, Forstmeier W, Schielzeth H, Mellenius H, Nam K, Bolund E et al. (2010a). The recombination landscape of the zebra finch Taeniopygia guttata genome. Genome Res 20: 485-495.

Backström N, Palkopoulou E, Qvarnström A, Ellegren H (2010b). No evidence for Z-chromosome rearrangements between the pied flycatcher and the collared flycatcher as judged by gene-based comparative genetic maps. Mol Ecol 19: 3394-3405.

Bennett JH, Oertel CR (1965). The approach to a random association of genotypes with random mating. J Theoret Biol 9: 67-76.

Bruford MW, Hanotte O, Brookfield JFY, Burke T (1998). Multilocus and single-locus DNA fingerprinting. In: Hoelzel AR (ed). Molecular Genetic Analysis of Populations: A Practical Approach. IRL Press: Oxford, UK, pp 225-269.

Brush $\mathrm{AH}$, Seifried $\mathrm{H}$ (1968). Pigmentation and feather structure in genetic variants of the Gouldian finch, Poephila gouldiae. The Auk 85: 416-430.

Carneiro M, Ferrand N, Nachman MW (2009). Recombination and speciation: Loci near centromeres are more differentiated than loci near telomeres between subspecies of the European rabbit (Oryctolagus cuniculus). Genetics 181: 593-606.

Christidis L (1986a). Chromosomal evolution within the family Estrildidae (Aves) I. The Poephilae. Genetica 71: 81-97.

Christidis L (1986b). Chromosomal evolution within the family Estrildidae (Aves) II. The Lonchurae. Genetica 71: 99-113.

Dawson D, Brekke P, Dos Remedios N, Horsburgh G (2015). A marker suitable for sex-typing birds from degraded samples. Conserv Genet Resour 7: 337-343.

Dawson DA (2007). Genomic Analysis of Passerine Birds Using Conserved Microsatellite Loci. University of Sheffield: Sheffield, UK.

Ducrest A-L, Keller L, Roulin A (2008). Pleiotropy in the melanocortin system, coloration and behavioural syndromes. Trends Ecol Evol 23: 502-510.

Evans S, Fidler M (2005). The Gouldian Finch. Indruss Productions: Queensland, Australia.

Green P, Falls K, Crooks S (1990). Documentation for CRI-MAP, Version 2.4. Washington University: St Louis, MO, USA.

Hooper DM, Price TD (2015). Rates of karyotypic evolution in Estrildid finches differ between island and continental clades. Evolution 69: 890-903.

Hugall AF, Stuart-Fox D (2012). Accelerated speciation in colour-polymorphic birds. Nature 485: 631-634.

Huynh LY, Maney DL, Thomas JW (2011). Chromosome-wide linkage disequilibrium caused by an inversion polymorphism in the white-throated sparrow (Zonotrichia albicollis). Heredity 106: 537-546.
Itoh Y, Kampf K, Arnold A (2006). Comparison of the chicken and zebra finch $Z$ chromosomes shows evolutionary rearrangements. Chromosome Res 14: 805-815.

Itoh Y, Kampf K, Balakrishnan CN, Arnold AP (2011). Karyotypic polymorphism of the zebra finch Z chromosome. Chromosoma 120: 255-264.

Jetz W, Thomas GH, Joy JB, Hartmann K, Mooers AO (2012). The global diversity of birds in space and time. Nature 491: 444-448.

Johnston SE, McEwan JC, Pickering NK, Kijas JW, Beraldi D, Pilkington JG et al. (2011). Genome-wide association mapping identifies the genetic basis of discrete and quantitative variation in sexual weaponry in a wild sheep population. $\mathrm{Mol} E \mathrm{col} 20$ : 2555-2566.

Kawakami T, Smeds L, Backström N, Husby A, Qvarnström A, Mugal CF et al. (2014). A high-density linkage map enables a second-generation collared flycatcher genome assembly and reveals the patterns of avian recombination rate variation and chromosomal evolution. Mol Ecol 23: 4035-4058.

Kim K-W, Zhang H, Horsburgh G, Bird S, Dawson D, Griffith S et al. (2015). Four-way development of microsatellite markers for the Gouldian finch (Erythrura gouldiae). Conserv Genet Resour 7: 899-907.

Küpper C, Stocks M, Risse JE, dos Remedios N, Farrell LL, McRae SB et al. (2016). A supergene determines highly divergent male reproductive morphs in the ruff. Nat Genet 48: 79-83.

Lamichhaney S, Fan G, Widemo F, Gunnarsson U, Thalmann DS, Hoeppner MP et al. (2016). Structural genomic changes underlie alternative reproductive strategies in the ruff (Philomachus pugnax). Nat Genet 48: 84-88.

Mank JE, Nam K, Ellegren H (2010). Faster-Z evolution is predominantly due to genetic drift. Mol Biol Evol 27: 661-670.

McKinnon JS, Pierotti MER (2010). Colour polymorphism and correlated characters: genetic mechanisms and evolution. $\mathrm{Mol}$ Ecol 19: 5101-5125.

Morton NE (1955). Sequenctial tests for the detection of linkage. Am J Hum Genet 7: 277-318.

Navarro A, Barton NH (2003). Chromosomal speciation and molecular divergenceaccelerated evolution in rearranged chromosomes. Science 300: 321-324.

Poelstra JW, Vijay N, Bossu CM, Lantz H, Ryll B, Muller I et al. (2014). The genomic landscape underlying phenotypic integrity in the face of gene flow in crows. Science 344: 1410-1414.

Pryke SR (2010). Sex chromosome linkage of mate preference and color signal maintains assortative mating between interbreeding finch morphs. Evolution 64: 1301-1310.

Pryke SR, Griffith SC (2006). Red dominates black: agonistic signalling among head morphs in the colour polymorphic Gouldian finch. Proc R Soc Ser B 273: 949-957.

Pryke SR, Griffith SC (2007). The relative role of male vs. female mate choice in maintaining assortative pairing among discrete colour morphs. Jf Evol Biol 20: 1512-1521.

Pryke SR, Griffith SC (2009a). Genetic incompatibility drives sex allocation and maternal investment in a polymorphic finch. Science 323: 1605-1607.

Pryke SR, Griffith SC (2009b). Postzygotic genetic incompatibility between sympatric color morphs. Evolution 63: 793-798.

Qvarnström A, Bailey RI (2008). Speciation through evolution of sex-linked genes. Heredity 102: 4-15.

Rieseberg LH (2001). Chromosomal rearrangements and speciation. Trends Ecol Evol 16: 351-358.

Sharlow ER, Paine CS, Babiarz L, Eisinger M, Shapiro S, Seiberg M (2000). The proteaseactivated receptor-2 upregulates keratinocyte phagocytosis. J Cell Sci 113: 3093-3101.

Singhal S, Leffler EM, Sannareddy K, Turner I, Venn O, Hooper DM et al. (2015). Stable recombination hotspots in birds. Science 350: 928-932.

Southern HN (1945). Polymorphism in Poephila gouldiae gould. J Genet 47: 51-57.

Stapley J, Birkhead TR, Burke T, Slate J (2010). Pronounced inter- and intrachromosomal variation in linkage disequilibrium across the zebra finch genome. Genome Res 20: 496-502.

Stump AD, Shoener JA, Costantini C, Sagnon NF, Besansky NJ (2005). Sex-linked differentiation between incipient species of Anopheles gambiae. Genetics 169: 1509-1519.

Thorneycroft HB (1966). Chromosomal polymorphism in the white-throated sparrow, Zonotrichia albicollis (Gmelin). Science 154: 1571-1572.

Tuttle EM (2003). Alternative reproductive strategies in the white-throated sparrow: behavioral and genetic evidence. Behav Ecol 14: 425-432.

Voorrips RE (2002). MapChart: software for the graphical presentation of linkage maps and QTLs. J Hered 93: 77-78.

Warren WC, Clayton DF, Ellegren H, Arnold AP, Hillier LW, Künstner A et al. (2010). The genome of a songbird. Nature 464: 757-762.

Wellenreuther M, Svensson El, Hansson B (2014). Sexual selection and genetic colour polymorphisms in animals. Mol Ecol 23: 5398-5414.

Zhang G, Li C, Li Q, Li B, Larkin DM, Lee C et al. (2014). Comparative genomics reveals insights into avian genome evolution and adaptation. Science 346: 1311-1320.

Supplementary Information accompanies this paper on Heredity website (http://www.nature.com/hdy) 